\title{
Beyond the standard model physics at RHIC in polarized pp collision
}

\author{
Jiro Murata \\ RIKEN, Wako 351-0198, Japan
}

\begin{abstract}
A polarized hadron collider experiment must have a great discovery potential for a search of physics beyond the standard model. Experimental data of various symmetry tests at RHIC are going to be obtained within a few years. The author developed a simulation tool, studying a sensitivity of hunting contact interaction at RHIC by measuring parity violating spin asymmetries.
\end{abstract}

\section{Introduction}

One of the most vital tasks of an experiment using a polarized hadron collider in a new energy region is the study of physics beyond the standard model. In the past few years, several theoretical works 11, 2, 3 have been devoted to the studies of the contact interaction (or, compositeness) 顿 and new gauge bosons $W^{\prime \pm}$ 河 and $Z^{\prime}[6]$. In these theoretical works, [7] it has been shown that RHIC can reach a similar sensitivity to that of the TEVATRON, due to its polarized beam.

The purpose of the present study is to explore the discovery potential for physics beyond the standard model from the experimental perspective. Considering the family pattern of quarks and leptons, compositeness is an fascinating idea. For there are no standard sub-quark models, model independent treatment using a compositeness scale $\Lambda$ is often used. We call such new generalized interaction as the contact interaction. One possible Feynmann diagram is shown in Fig.1. For any kind of an new interaction with energy scale $\Lambda$ can be taken into account, for example, new gauge boson exchange between "standard" quarks can be understood as a kind of the contact interaction. $\Lambda$ is a model-independent scale parameter of the contact interaction, defined as $F\left(Q^{2}\right)=\left(1+Q^{2} / \Lambda^{2}\right)^{-1}$, where $F\left(Q^{2}\right)$ is a 'form factor' of the quark and lepton. Therefore, sometimes one interpret the physical meanings of the the contact interaction as quark (lepton) compositeness. However, the contact interaction has general formalism, so any kind of new interaction can be roughly included.

\section{Simulation Procedure using PYTHIA+POLBY}

In order to study physics sensitivity and to determine a search window, simulation studies with an event generator are indispensable. Several non-standard scenarios can be examined using PYTHIA. The contact interaction can be also examined through it. However, as with all other subprocesses, PYTHIA includes only helicity-averaged cross sections for the contact interaction. The author developed a plug-in program 'POLBY' for PYTHIA by which hadronic spin asymmetries for Drell-Yan and quark-scattering process can be examined. Helicity-selected matrix 
elements have been theoretically obtained in LO not only for the standard model but also for the contact interaction. In case of Drell-Yan process, corresponding matrix elements were obtained from Virey's calculation for DIS by crossing [8]. A weighted mean method is applied in order to extract spin asymmetries using "unpolarized" event generator PYTHIA. A weight factor is calculated for each generated event, then its mean value of the weight factor distribution gives us the final spin asymmetries. First, partonic cross section $\hat{\sigma}$ can be obtained at a kinematics region by summing up the matrix elements for all the possible interactions. In order to estimate the hadronic spin asymmetries, we need hadronic cross sections $\sigma^{h_{1} h_{2}}$ with helicity $h_{1}, h_{2}$ selection. They can be obtained by combining with polarized quark distribution functions $q^{ \pm}$. For example, $\sigma^{++}$can be obtained as;

$$
\sigma^{++}=\hat{\sigma}^{++} q_{1}^{+} q_{2}^{+}+\hat{\sigma}^{--} q_{1}^{-} q_{2}^{-}+\hat{\sigma}^{+-} q_{1}^{+} q_{2}^{-}+\hat{\sigma}^{-+} q_{1}^{-} q_{2}^{+} .
$$

Using the hadronic cross sections, we can estimate the weight factor in combinations between different spin selected hadronic cross sections. For single spin asymmetry

$$
A_{L}=\frac{\sigma^{-}-\sigma^{+}}{\sigma^{-}+\sigma^{+}}=\frac{\sigma^{--}+\sigma^{-+}-\sigma^{++}-\sigma^{+-}}{\sigma^{--}+\sigma^{-+}+\sigma^{++}+\sigma^{+-}}
$$

and parity violating double spin asymmetry

$$
A_{L L}^{P V}=\frac{\sigma^{--}-\sigma^{++}}{\sigma^{--}+\sigma^{++}}
$$

corresponding weight factors can be obtained by making the same cross section ratio in event-by-event. The distribution of the weight factor depends on the quark distribution functions, therefore, the process of making a mean value on these distribution implies an Monte-Carlo integration over the quark distribution functions. There are two free parameters in the matrix elements for the contact interaction. First one is the scale parameter $\Lambda$ and the second one is a sign of the interference between the standard model and the contact interaction. 
Beyond the standard model physics at RHIC in polarized pp collision...

\section{Drell-Yan process}

Parity violating spin asymmetries are examined using PYTHIA+POLBY. In PYTHIA, Drell-Yan events can be generated in single subprocess, which include the standard model $\gamma^{*} / Z^{0}$ exchange and also the contact interaction with its scale $\Lambda$. Fig. 2 shows the results on Drell-Yan yield with selections of beam energies $(\sqrt{s}$ $=500,750,1000 \mathrm{GeV}$ ) and the scale $\Lambda$. Here, GS-A polarized parton distribution function [9] is used. At any beam energy, excess from the standard model are observed when we require $\Lambda=1 \mathrm{TeV}$, however, the excess is negligible if $\Lambda \geq 2$ $\mathrm{TeV}$. Considering the percent-level systematic error in a cross section measurement, observation of the cross section excess is hopeless. In Drell-Yan process, contribution from the pure contact interaction is very small because of its $\Lambda$ dependence of $\Lambda^{-4}$, on the other hand, the interference terms between the contact interaction and $Z^{0}$, $\gamma^{*}$ have $\Lambda^{-2}$ dependence.

On the other hand, a polarized experiment has a great advantage because of its systematic error cancellation. Simulation results using PYTHIA+POLBY on the parity violating single spin asymmetry $A_{L}$ are shown in Fig. 3. Pure $Z^{0}$ exchange and $\gamma^{*}-Z^{0}$ interference contribute on the parity violation on the standard model. We can see the large deviation from the standard model if we include the contact interaction. Dominant contribution on the parity violation is the interference term between the contact interaction and the standard model. In Fig.3, we can see a clear deviation from the standard model even at $\Lambda=5 \mathrm{TeV}$, however, the experimental statistical error have to be also large. Because of its relatively small cross section, required integrated luminosity is large as shown in Fig. A. Considering the RHIC luminosity of $800 \mathrm{pb}^{-1}$ at $\sqrt{s}=500 \mathrm{GeV}$, our sensitivity is limited around $\Lambda \sim 1$ $\mathrm{TeV}$, which is lower than the already established limits in $e^{+} e^{-}$collisions for the electron-quark contact interaction $(\Lambda>5.4 \mathrm{TeV})$ by ALEPH and in $\bar{p} p$ collisions for the muon-quark contact interaction $(\Lambda>2.9 \mathrm{TeV})$ by $\mathrm{CDF}$ [10].

\section{Inclusive Jet production process}

Although Drell-Yan process is simple and clean, we need to examine other process, which has large production cross section because of the small yield on DrellYan process. Parity violation in the inclusive jet production process has already examined by J.-M. Virey theoretically [7]. The jet production cross section is shown in Fig. 5 as functions of jet $P_{T}$. Lower $P_{T}$ region is dominated by gluon-related processes, on the other hand, higher $P_{T}$ region is dominated by quark-quark scattering. The contact interaction couples to leptons and quarks, but not to gluons in the current model. It means that we do not think gluon is a composite particle. Therefore, sensitive $P_{T}$ region of the contact interaction must be limited to the higher $P_{T}$ region. In case of Drell-Yan process, all the interactions we need to consider, are the standard model $\gamma^{*} / Z^{0}$, the contact interaction, and their interferences. On the other hand, we need to take into consideration QCD, $\gamma^{*}, Z^{0}, W^{ \pm}$, the contact interaction, and their complex interference terms for the jet production. In PYTHIA, above processes must be generated as independent subprocesses. This restriction 
cause severe problem when we consider the interference terms between different subprocesses in PYTHIA. It is because there cannot exist interference effects if we distinguish the subprocess. In case of Drell-Yan process, we can use single subprocess, therefore, the interference effects can be automatically included. In order to restore quantum mechanics in the jet production, a special treatment is required. In POLBY, a hadronic spin asymmetry $A$ is estimated using event-by-event asymmetry weight factor $W(A)$;

$$
A=\frac{\sum_{i=e v e n t} W^{i}(A)}{\sum_{i=\text { event }}} .
$$

Here, the "events" are generated by PYTHIA subprocess. The "interference correction" can be done by

$$
W^{i}(A) \rightarrow W^{i}(A) \times \frac{\sum_{\text {process }=Q C D, W e a k, E M, C T} \sigma^{\text {process }}}{\sigma^{Q C D}}
$$

with Eq. . Then if we use pure QCD subprocess for the PYTHIA event generation, contribution from other processes on the asymmetry can be taken into account by summing up over all the processes. Fig.6 shows a sample distribution of $W(A)$ before/after the interference correction of Eq. The mean value of the $W(A)$ distribution shows the final hadronic spin asymmetry. By the operation Eq. 5 . $W(A)$ distribution is distorted and we can get the corrected asymmetry from the distorted distribution. By using Eq.5, we can estimate accurate asymmetries. For an example, parity violation around Jacobean peak is examined. In Fig.7, we can confirm the large contribution from the interference term on the parity violation around the Jacobean peak. Subprocess-separated plot in Fig.] shows that $q \bar{q}^{\prime} \rightarrow q \bar{q}^{\prime}$ subprocess dominates the parity violation at the $\mathrm{W}$ peak. Fig.8 shows a preliminary results, which can be compared with the theoretical calculation [7]. Considering the difference on the pseudo rapidity cut and binding, the results meets well with the calculation by Virey. Using these results, discovery potential at RHIC can be explored. In Fig 9 , required integrated luminosities are plotted as functions of $\Lambda$. Here combined results from the present study at lower $\Lambda$ region and that from Virey at higher $\Lambda$ region 11] are shown. It is shown that we can reach more than $3 \mathrm{TeV}$ sensitivity, which is higher than the current limit of $2.7 \mathrm{TeV}$ reported by $\mathrm{D} 0$ for the quark-quark contact interaction, 10 with the current RHIC plan. It is also shown that the sensitivity limit can be more than $6 \mathrm{TeV}$ using the RHIC upgrade plan. We can calculate many combinations of parity violating spin asymmetries using PYTHIA+POLBY with many realistic experimental conditions. For the procedure using weighted mean can be understood as a kind of Monte-Carlo integration, the calculation speed is very fast. Therefore this is a strong tool to examine an experimental sensitivity and also to make a standard model reference after getting experimental data.

However, in order to use it as a standard model reference, NLO calculation is indispensable. NLO effect is difficult to take into an event generator. The situation is similar to the treatment of the interference. One large assumption for the interference correction is that, pure QCD process (generated events) and other processes 
must have their same final states. In principle, NLO correction using similar cross section correction like;

$$
W^{i}(A) \rightarrow W^{i}(A) \times \frac{\sum_{\text {process }=N L O+L O} \sigma^{\text {process }}}{\sigma^{L O}}
$$

should be able to be applied. Theoretical calculations in NLO are now under progress [12]. We should and will be able to be ready to examine experimental data from RHIC in near future.

\section{Acknowledgement}

Many thanks are due to J.-M. Virey and J. Soffer for the continuous help from theoretical side, especially for the treatment of the matrix elements. The author is also grateful

to G. Bunce, N. Saito and M. Tannenbaum for the advises on the experimental aspects and on the simulation formalism.

\section{References}

[1] F. E.Paige and M. J.Tannenbaum, CBA Newsletter No.4 (1983) as quoted by R. Ruckl, J. de Physique 46 (1985) C2-55.

[2] C. Bourrely et. al. Phys. Rep. 177, 319 (1989).; Nucl. Phys. 36172 (1991).

[3] P. Taxil, Nuo. Cim. Vol. 16, N 11, (1993).

[4] E. J.Eichten et. al. Phys. Rev. Lett. 50811 (1983).; Rev. Mod. Phys. 56 No.4 (1984).

[5] P. Taxil and J.-M. Virey, Phys. Lett. B 404302 (1997).

[6] P. Taxil and J.-M. Virey, Phys. Lett. B 441376 (1998).; B 383355 (1996)

[7] P. Taxil and J.-M. Virey, Phys. Lett. B 364181 (1995).; Phys. Rev. D 55, 4408 (1997); J.-M. Virey, hep-ph/9707470

[8] J.-M. Virey, Eur. Phys. J. C 8, 283 (1999).

[9] T. Gehrmann and W. J. Stirling, Phys. Rev. D 53, 6100 (1996).

[10] Particle Data Group, Eur. Phys. J. C 15 (2000)

[11] P. Taxil and J.-M. Virey, hep-ph/0109094

[12] J. Ellis, S. Moretti and D. A.Ross, hep-ph/0102340

\section{$5 \quad$ Future perspectives}




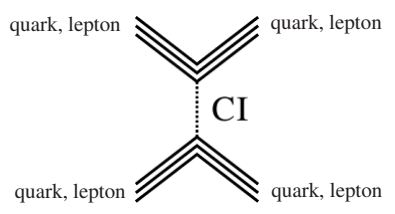

Fig. 1. Possible Feynmann Diagram of the contact interaction with energy scale $\Lambda$.

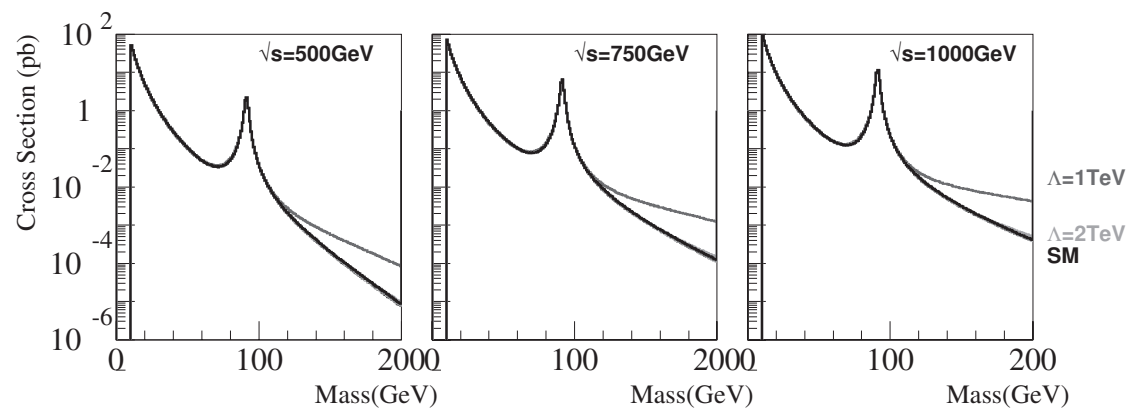

Fig. 2. Cross section of Drell-Yan process is shown including the contact interaction. 
Beyond the standard model physics at RHIC in polarized pp collision...

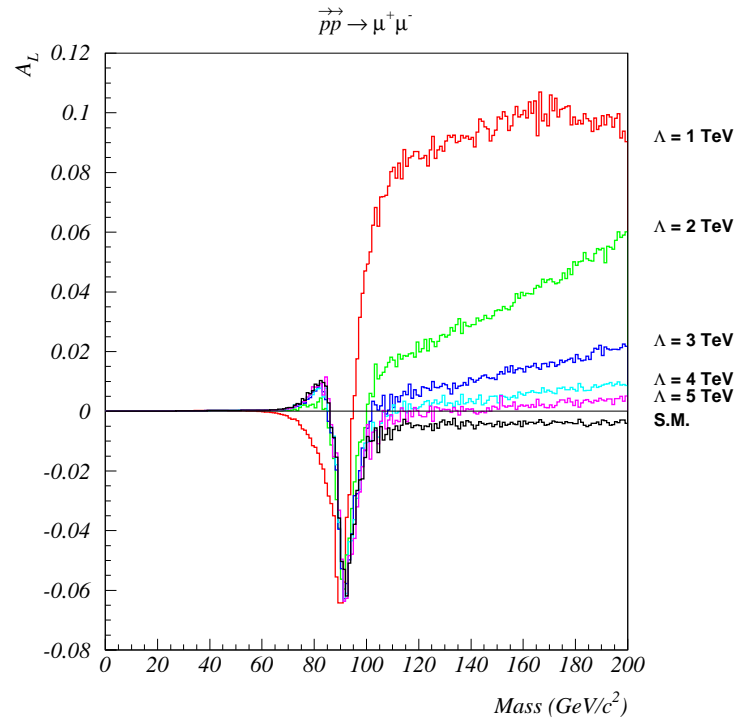

Fig. 3. Single spin asymmetries $A_{L}$ are drawn as functions of Drell-Yan mass with selections of $\Lambda$.

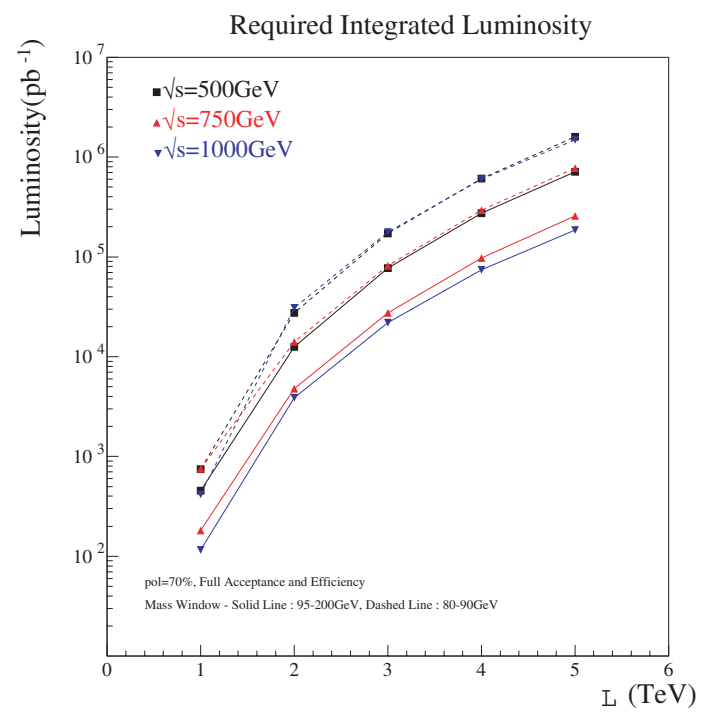

Fig. 4. Required integrated luminosity as functions of $\Lambda$ is shown. 


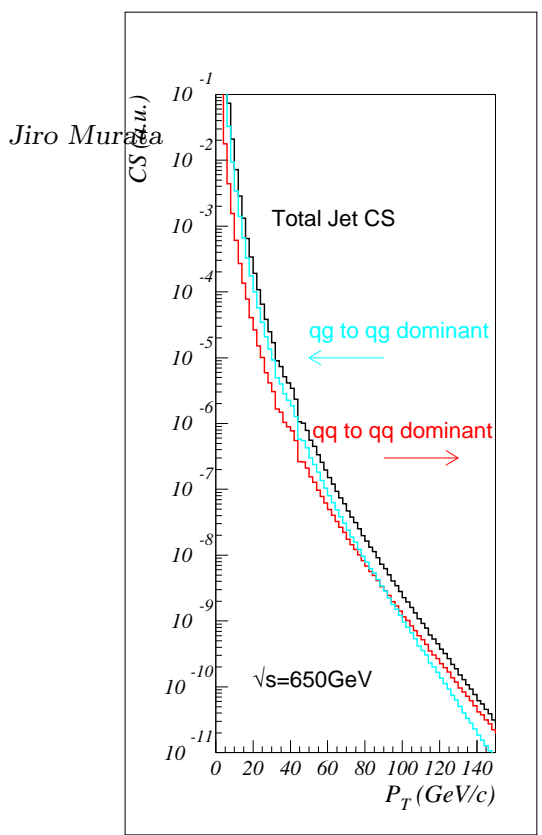

Fig. 5. Jet production cross section is shown. At high Pt region, quark-quark scattering is dominant. Small bump around $M_{W} / 2$ corresponds Jacobean Peak

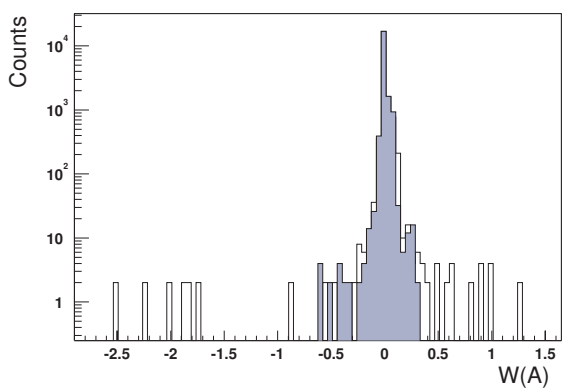

Fig. 6. Sample plot of the weight factor. Hatched(Blank) histogram shows before(after) the interference correction. 
Beyond the standard model physics at RHIC in polarized pp collision...
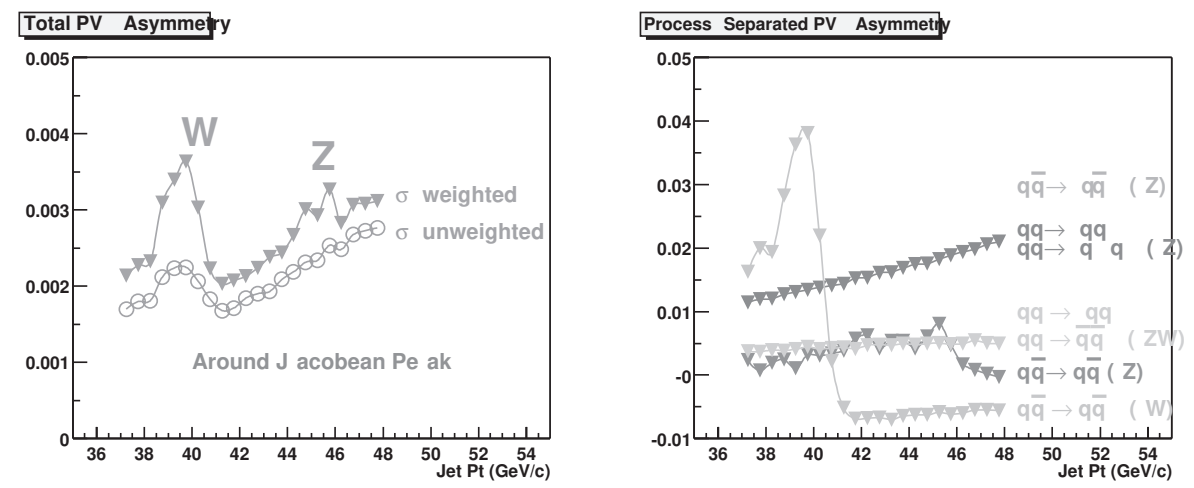

Fig. 7. Fine structure of the Jacobean peak on parity violating spin asymmetries.

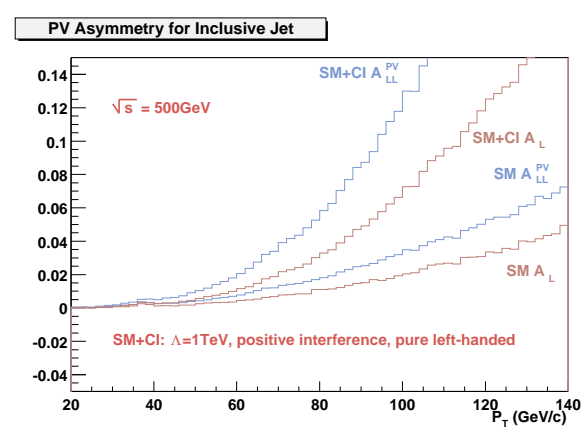

Fig. 8. Expected parity violating spin asymmetries. 
Jiro Murata

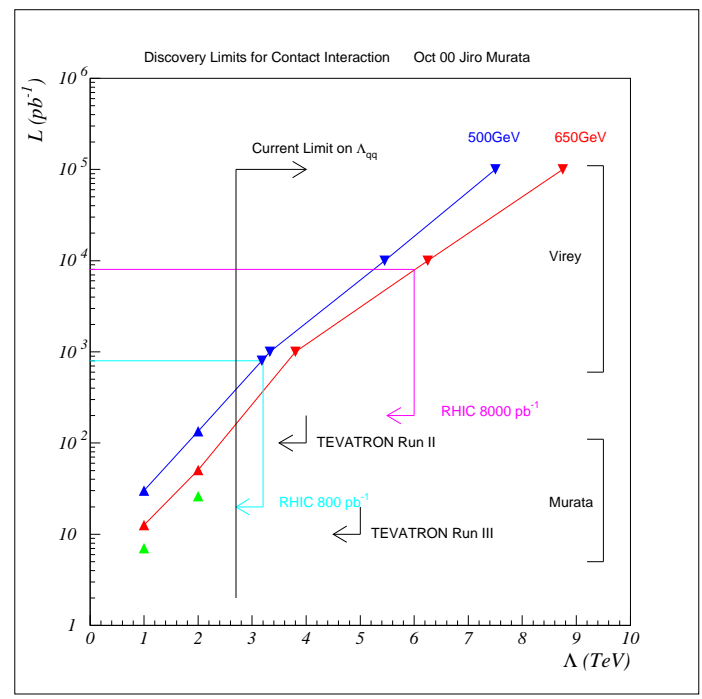

Fig. 9. Required integrated luminosities are plotted as functions of $\Lambda$. 